\title{
Persistent quantum walks: Dynamic phases and diverging timescales
}

\author{
Suchetana Mukhopadhyay (1) and Parongama Sen (1) \\ Department of Physics, University of Calcutta, Kolkata 700009, India
}

(Received 31 October 2019; accepted 6 March 2020; published 2 April 2020)

\begin{abstract}
A discrete-time quantum walk is considered in which the step lengths are chosen to be either 1 or 2 with the additional feature that the walker is persistent with a probability $p$. This implies that with probability $p$, the walker repeats the step length taken in the previous step and is otherwise antipersistent. We estimate the probability $P(x, t)$ that the walker is at $x$ at time $t$ and the first two moments. Asymptotically, $\left\langle x^{2}\right\rangle \propto t^{\nu}$ for all $p$. For the extreme limits $p=0$ and 1 , the walk is known to show ballistic behavior, i.e., $v=2$. For both $p=0^{+}$and $1-p=0^{+}$we find that the scaling behavior changes discontinuously with $v=1$ for $p=0^{+}$and $v=1.5$ for $p \rightarrow 1$. For $0<p<p_{c}$, v varies as $1+0.5[1-\exp (-\gamma p)]$ with $\gamma \approx 14.42$ and $p_{c}$ close to 0.33 . For $p_{c}<p<1, v=3 / 2$. Close to $p=0,1$, the scaling behavior shows a crossover in time. Associated with this crossover, two diverging timescales varying as $1 / p$ and $1 /(1-p)$ close to $p=0$ and $p=1$, respectively, are detected. Using a different scheme in which the antipersistence behavior is suppressed, one gets $v=3 / 2$ for the entire region $0<p<1$.
\end{abstract}

DOI: 10.1103/PhysRevResearch.2.023002

\section{INTRODUCTION}

Discrete-time quantum walks (DTQW's), first introduced by Aharonov et al. [1], are random walks where a coin degree of freedom is introduced which determines the translation of the walker. Quantum interference in such walks leads to the position $x$ of the walker scaling as $\left\langle x^{2}\right\rangle \propto t^{2}$ with time $t$, indicating a quadratically faster spread compared to the classical random walk.

The introduction of randomness or disorder in quantum walks has been demonstrated to modify its scaling behavior significantly. Disorder can be incorporated in various ways, and several studies in recent years have focused on the modifications they impart and how they may turn out to be useful [2]. Disorder introduced through interaction with the external environment or the presence of broken links in position space tends to slow down the quantum walk and leads to localization $[3,4]$. Such localization effects were first studied by Anderson [5] in the context of electron localization in a disordered lattice. Anderson localization-type behavior has been observed experimentally by introducing static (positional) disorder in a quantum walk on a homogeneous lattice [6]. Static disorder can be contrasted with dynamic disorder or decoherence [4] that transforms the quantum walk to the classical equivalent (diffusive scaling; $\left\langle x^{2}\right\rangle-\langle x\rangle^{2} \propto t$ ).

Dynamic disorder is usually introduced through the operations controlling the evolution of the quantum walk, such as by using decoherent coins $[7,8]$. It is also possible to incorporate dynamic disorder by relaxing the standard assumption of a

Published by the American Physical Society under the terms of the Creative Commons Attribution 4.0 International license. Further distribution of this work must maintain attribution to the author(s) and the published article's title, journal citation, and DOI. constant displacement at each time step and allowing longer steps to be chosen randomly, as in Refs. [9-11] where the scaling $\left\langle x^{2}\right\rangle \propto t^{\frac{3}{2}}$ was found.

In the present work we introduce the concept of persistence in the quantum walk. In a classical random walk, persistence implies that the walker continues in the direction taken in the previous step, making it non-Markovian. In the quantum walk, in order to introduce the idea of persistence, we allow the walker to take different step lengths at each step and remember the step length chosen in the preceding step with a certain probability. This provides a simple way to study the effect of short-term memory in the long-ranged walk. The probability distribution of the position of the walker and its first and second moments are evaluated and the results compared with the classical walk and the quantum walk without disorder.

Memory effects in quantum walks have generated a lot of interest recently, as evidenced by quite a few works [12-16]. Both short-term and long-term memory have been considered but in a very different manner. In particular, in the case of the so-called elephant quantum walk [15], where the walker has infinite memory as well as time-dependent step lengths, the variance was found to scale as $t^{3}$. To the best of our knowledge, the persistence in quantum walks, the way it has been incorporated in the present work, has not been considered before.

In the next section we introduce the quantum walk and the exact way the concept of persistence has been used. In Sec. III, the results have been presented. Section IV includes a summary of the results and a detailed discussion on the implications and the insight developed through the study.

\section{THE PERSISTENT QUANTUM WALK}

In the simple DTQW in one dimension, the walker can occupy discrete, equispaced sites $x$ on the real line and takes a step at unit time intervals. In addition to the position, the 
walker is assigned a second degree of freedom by means of a coin state [either left $(|L\rangle)$ or right $(|R\rangle)]$. The state of the walker is described by the following two-component vector expressing probability amplitudes for the coin states:

$$
|\psi(x, t)\rangle=\langle x \mid \psi(t)\rangle=\left[\begin{array}{l}
a(x, t) \\
b(x, t)
\end{array}\right] .
$$

The occupation probability of the site $x$ at time step $t$ is given by $P(x, t)=|\langle x \mid \psi(t)\rangle|^{2}=|a(x, t)|^{2}+|b(x, t)|^{2}$ with the total probability equal to 1 at each time step. A step in the quantum walk consists of a rotation in the coin space followed by a translation. A standard choice for this rotation operator is the Hadamard coin $H$, given by

$$
H=\frac{1}{\sqrt{2}}\left[\begin{array}{rr}
1 & 1 \\
1 & -1
\end{array}\right] .
$$

Instead of defining the step length to be a constant $l$, we allow it to be chosen from a binary distribution, $l(t)=\{1,2\}$, at any given time $t$. The conditional translation operator at a time $t$ is then written as

$$
\begin{aligned}
T(t)= & |R\rangle\left\langle R\left|\otimes \sum_{x}\right| x+l(t)\right\rangle\langle x+l(t)| \\
& +|L\rangle\left\langle L\left|\otimes \sum_{x}\right| x-l(t)\right\rangle\langle x-l(t)| .
\end{aligned}
$$

Allowing for a nonunique step length in this way enables one to study the phenomenon of persistence by considering the tendency to adopt the step length used in the previous time step. This choice is made in two ways, outlined under two different schemes, I and II. In each scheme, at $t=0$, the step length $l(0)$ is chosen to be 1 or 2 with equal probability. In Scheme I, at any later time $t \neq 0$, the walker either chooses the same step length as in the previous time step (persistent) and otherwise necessarily chooses the other step length (antipersistent). In Scheme II, the walker is persistent with a probability $p$, but this time with probability $(1-p)$, either of the step lengths $l=1$ and $l=2$ are chosen, with probability $q$ and $(1-q)$, respectively.

For both schemes the walker is initialized with $a(x, 0)=$ $b(x, 0)=\frac{1}{\sqrt{2}} \delta_{x, 0}$, which gives an asymmetric probability distribution profile in the absence of disorder. The walk is evolved for 20000 time steps for all parameter values. We investigate how the occupation probability and moments depend on the parameter(s) used in the two schemes. All results are averaged over 4000 configurations.

\section{RESULTS}

\section{A. Scheme I}

In this subsection we present the results for the first scheme considered. The walker here chooses the step length taken in the previous step with probability $p$ and with probability $(1-p)$ it chooses strictly the other length. The latter case thus corresponds to an antipersistent choice.

\section{Probability distribution}

When $p=0$, steps of length $l=1$ and 2 are taken alternately, with a possible sequence of steps given by $1,2,1,2,1 \ldots$, etc. The walk clearly has periodicity 2 , and there is no randomness in the choice of steps. This particular walk has already been studied in Ref. [9] and was found to have the same scaling behavior as the ordinary quantum walk. The probability distribution resembles an overlap of distributions obtained for the ordinary walks with $l=1$ and 2 [9].

On the other hand, when $p=1$, the walk becomes deterministic with a unique step length and thus identical to the usual quantum walk. The result for the point $p=0.5$ can also be easily guessed. Here essentially the step lengths 1 and 2 are being chosen with equal probability at each step. Hence it belongs to the class of models studied in Ref. [9].

As $p$ is increased even slightly from zero, the distribution $P(x, t)$ exhibits a peak centered at the origin in addition to two ballistic peaks. Such a central peak is not present in the ordinary quantum walk and neither in the binary walk without randomness. However, in presence of disorder and decoherence, such peaks indeed appear, when localization of the quantum walker takes place.

The ballistic peaks are signatures of the quantum nature of the walk and are in general asymmetric in height, reflecting the asymmetry of the pure quantum walk. As $p$ is increased, the ballistic peaks are seen to increase in height, while the central peak goes down. This can be interpreted as an increase in delocalization with increasing probability of the walker to be persistent, as the walker approaches the standard case of a fixed step length. Even for $p$ very close to 1, however, the central peak does not disappear. Exactly at $p=1$, the familiar distribution of the quantum walk is recovered, as expected.

Plotting $t^{\gamma} P(x, t)$ against the scaled variable $x / t^{\gamma}$, we observe data collapse for four different time steps, with $\gamma=0.5$ for the central peak and $\gamma=1.0$ for the ballistic peaks. We conclude that $P(x, t)$ exhibits two distinct scaling behaviors: The centrally peaked part scales as $x \propto \sqrt{t}$, like the classical walk, while the ballistic peaks scale like the ordinary quantum walk, $x \propto t$. These results are true for the entire region $0<$ $p<1$ as shown in Fig. 1 .

\section{Scaling of the moments}

We next present the results for the first two moments, $\langle x\rangle$ and $\left\langle x^{2}\right\rangle$, of the probability distribution.

In the limiting cases of $p=0$ and $p=1$, the walk reduces to an ordinary quantum walk such that $\langle x\rangle \propto t$ and $\left\langle x^{2}\right\rangle \propto t^{2}$. Interestingly, when $p$ deviates from zero or 1 , even by the smallest amount, we note that the asymptotic variations of the moments are significantly changed. The first moments are plotted in Fig. 2 for a few $p$ values, showing that the asymptotic exponent decreases to very small values for $p=$ $0^{+}$and increases up to a value close to $1 / 2$ at larger $p$ values.

We plot the second moments in Fig. 3 for a few values of $p$. Usually it is the variance which is used to characterize the walk. However, we note here that, asymptotically, $\left\langle x^{2}\right\rangle$ is larger than $\langle x\rangle^{2}$ by at least two orders of magnitude. Hence it suffices to consider how $\left\langle x^{2}\right\rangle$ behaves instead of the variance $\left\langle x^{2}\right\rangle-\langle x\rangle^{2}$. In the following, we study the behavior of the second moment in detail.

We note two things from Fig. 3; first, for $p$ values close to zero, there is a distinct change in the behavior of the moments with time; initially it has a fast growth but becomes much 

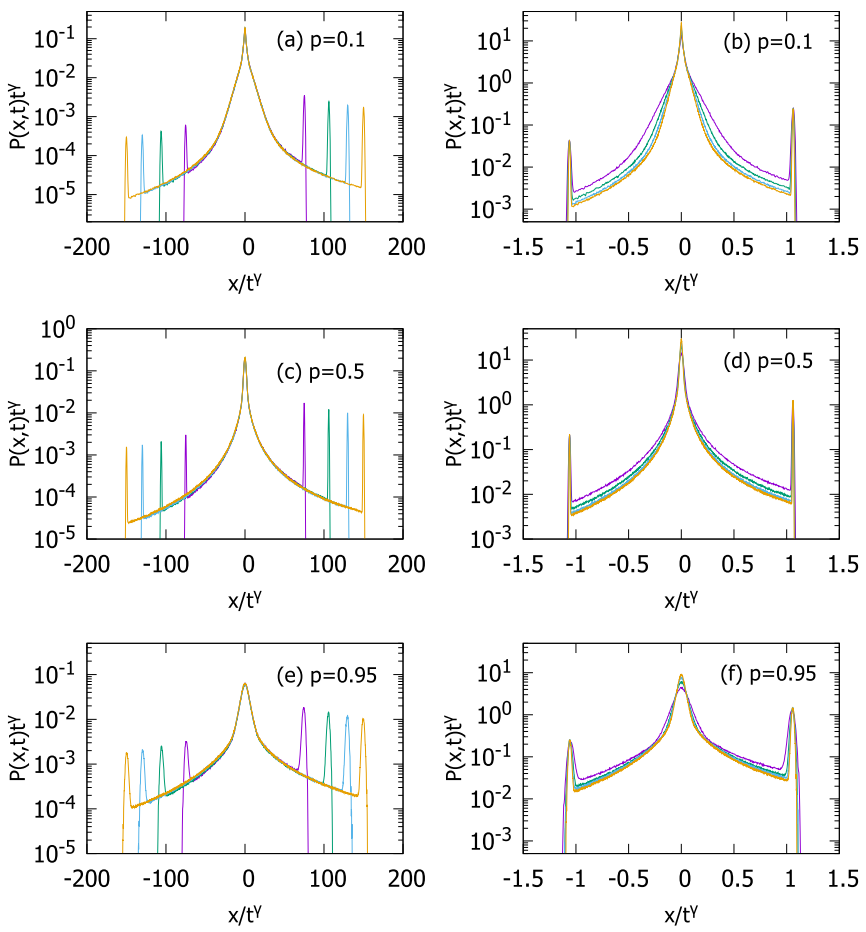

FIG. 1. Scheme I : Data collapse of rescaled $P(x, t)$ using $\gamma=$ 0.5 (left column) and $\gamma=1.0$ (right column). $\gamma=1$ collapse is less sharp.

slower later. Second, the asymptotic variation is significantly dependent on $p$. The curves can be fitted to piecewise powerlaw forms; for values of $p$ close to 1 , the asymptotic value of the corresponding exponent is 1.5 . For $p \rightarrow 0^{+}$, the variation is like $\left\langle x^{2}\right\rangle \propto t$. This is a drastic change from the behavior when $p$ is exactly zero.

We probe the $p \rightarrow 0^{+}$region in more detail as we have the most significant change in the asymptotic exponent value here. In the inset of Fig. $4,\left\langle x^{2}\right\rangle$ is plotted against $t$ and it clearly shows a variation compatible with $t^{2}$ for a long time

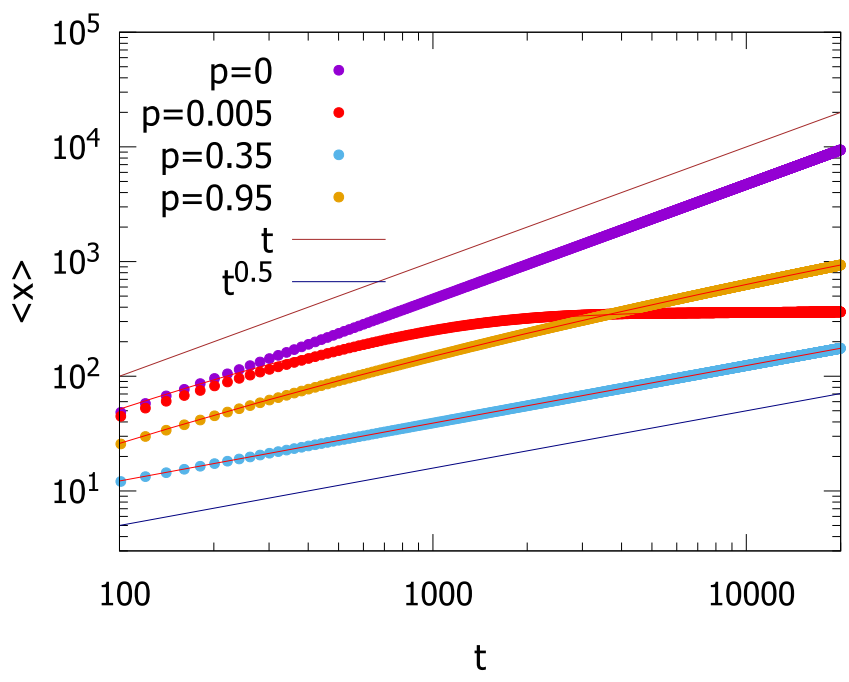

FIG. 2. Scheme I: The first moments for four $p$ values show significant change in behavior as $p$ is varied.

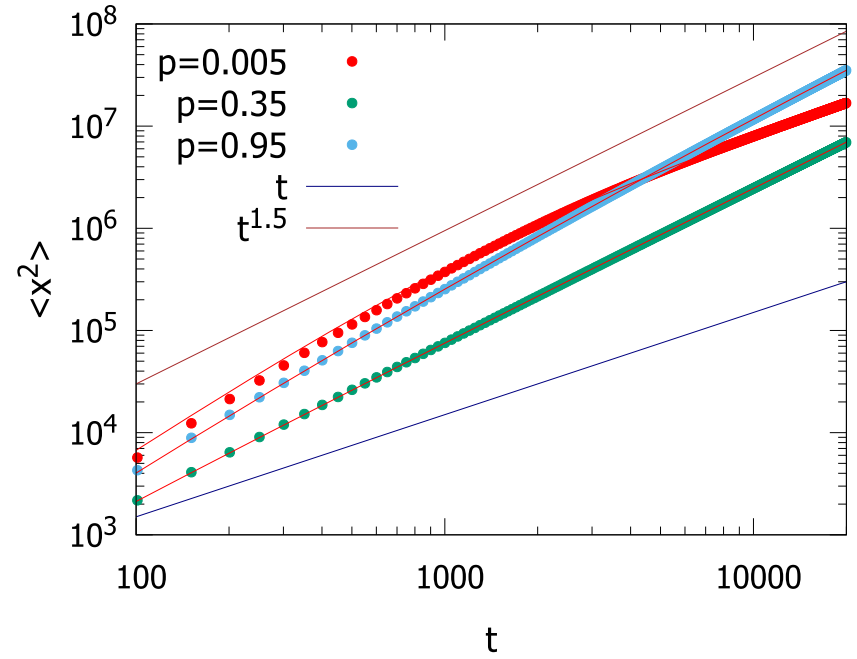

FIG. 3. Scheme I: The second moment for three $p$ values. The continuous lines are best-fit curves obtained using Eq. (5) with fitting parameters $\alpha^{\prime}=1.379,0.81,1.81$ and $\beta^{\prime}=0.0011,0.382,0.066$ for $p=0.005,0.35$, and 0.95 , respectively.

before deviating to a slower increase. The deviation occurs at larger values of time as $p$ approaches zero. Plotting $\left\langle x^{2}\right\rangle / t^{2}$ against the scaling variable $p t$ for several values of $p$ very close to zero, we obtain a very good collapse (Fig. 4) from which we claim $\left\langle x^{2}\right\rangle \propto t^{2} f(p t)$. It is clear from Fig. 4 that $f(z)$ fairly a constant for $z<1$. For $z \geqslant 1$, one can fit $f(z)$ to the form

$$
f(z)=1 /\left(\alpha+\beta z^{\mu}\right)
$$

to a great degree of accuracy with $\alpha \approx 1.7, \beta \approx 0.2$, and $\mu \approx$ 1. From this it can be concluded that a crossover occurs at $p t \approx 1$ and there is a diverging timescale $\tau$ varying inversely with $p$. On the other hand, the asymptotic variation is $\left\langle x^{2}\right\rangle \propto t$.

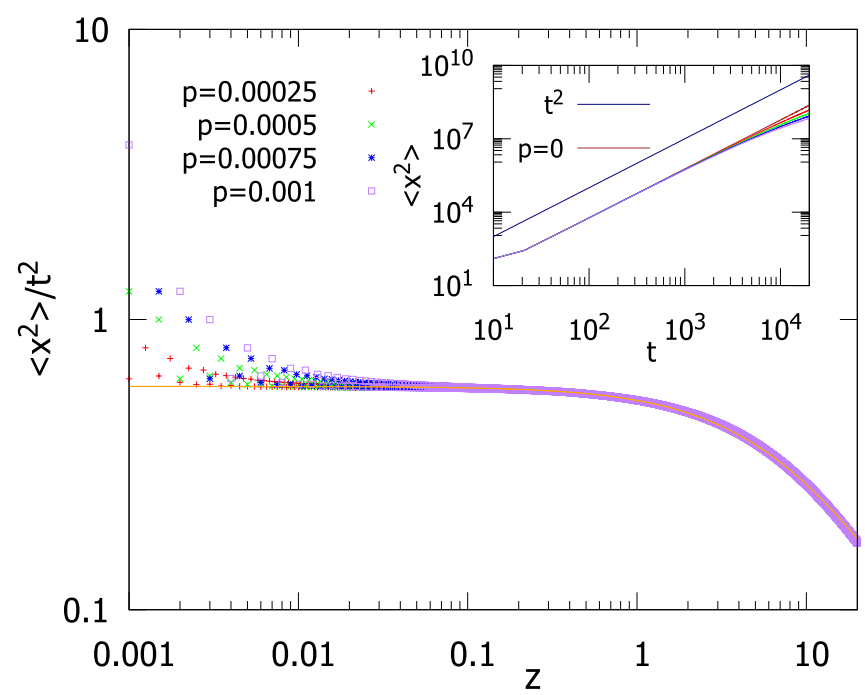

FIG. 4. Scheme I: Data collapse of the second moment of the distribution $P(x, t)$ obtained on plotting $\left\langle x^{2}\right\rangle / t^{2}$ against the scaling variable $z=p t$. The solid line is a best-fit line drawn using Eq. (4). Inset shows the unscaled data $\left\langle x^{2}\right\rangle$ against time $t$. The data for $p=0$ and a curve with quadratic variation are shown for comparison. 


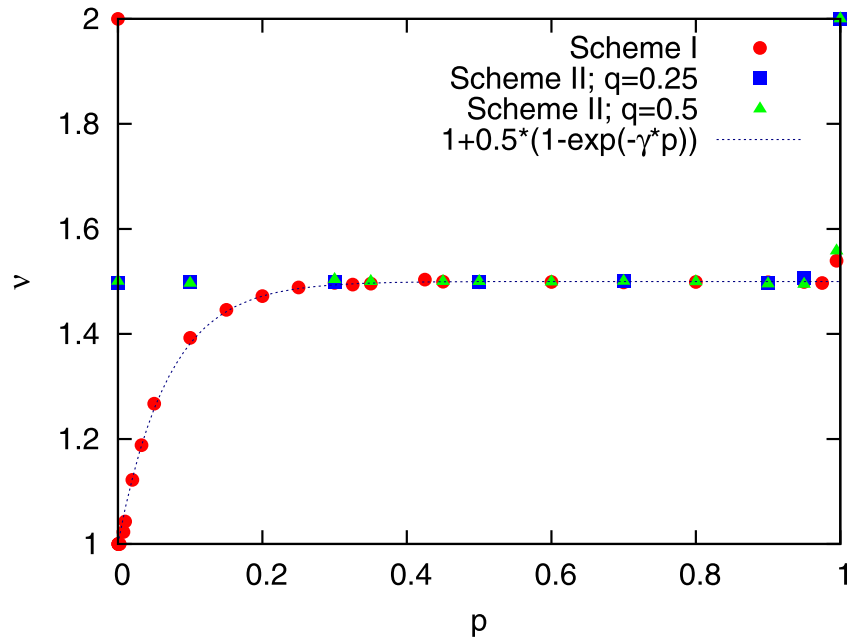

FIG. 5. Variation of the effective scaling exponent $v$ with $p$ for Scheme I and Scheme II. The best-fit curve for $v$ obtained in Scheme $\mathrm{I}$ is also shown (see text for details).

Thus the crossover time marks the transition to the asymptotic behavior.

As $p$ is made larger, the crossover occurs at smaller times and the exponent is extracted from fitting the second moment directly to the empirical form valid for later time regimes [as obtained for the $p \rightarrow 0^{+}$case, Eq. (4)],

$$
\left\langle x^{2}\right\rangle=t^{2} /\left(\alpha^{\prime}+\beta^{\prime} t^{2-v}\right),
$$

such that asymptotically, $\left\langle x^{2}\right\rangle \propto t^{\nu}$. The best-fit curves using the above form plotted for the data shown in Fig. 3 show excellent agreement. $\alpha^{\prime}$ varies nonmonotonically with $p$ as it drops sharply from $\sim 1.7$ at $p=0^{+}$. Up to $p \approx 0.33$, its value remains negligible, indicating pure power law behavior over the entire time regime with an exponent $v \neq 2 . \alpha^{\prime}$ increases again continuously to attain a value $\sim 1.7$ close to $p=1$. $\beta^{\prime}$ is zero at $p=0$ and $p=1$ as expected and takes a positive value $<0.5$ elsewhere.

In Fig. 5, the asymptotic exponent $v$ is plotted as a function of $p$ and it can be fit to the form

$$
v=1+0.5[1-\exp (-\gamma p)],
$$

with $\gamma=14.42 \pm 0.5$. The large value of $\gamma$ indicates that although there is a continuous variation of $v$, it reaches a value close to 1.5 for a fairly small value of $p$. In fact, $p=0.5$ corresponds to a purely random case for which the exponent was found to be $3 / 2$ [9]. Hence we conjecture that $v$ actually attains the value $3 / 2$ beyond a value of $p=p_{c}$ which, from inspection of Fig. 5, is close to 0.33 . We thus conclude that the above form of $v$ is valid up to $p_{c}$.

A similar crossover is noted close to $p=1$. The data for $\left\langle x^{2}\right\rangle$ against $t$ are plotted for $p$ very close to unity in Fig. 6. The overall variation agrees well with a power-law behavior with an exponent close to 2 for the range of time studied, although it shows signs of deviation from a perfect power law. On plotting $\left\langle x^{2}\right\rangle / t^{2}$ against $z^{\prime}=(1-p) t$ for several $p$ values very close to one, we obtain a good collapse. Till a value $z^{\prime} \approx 0.1$, the scaling function is fairly a constant. This corresponds to the early time regime where $\left\langle x^{2}\right\rangle \propto t^{2}$. The deviation from

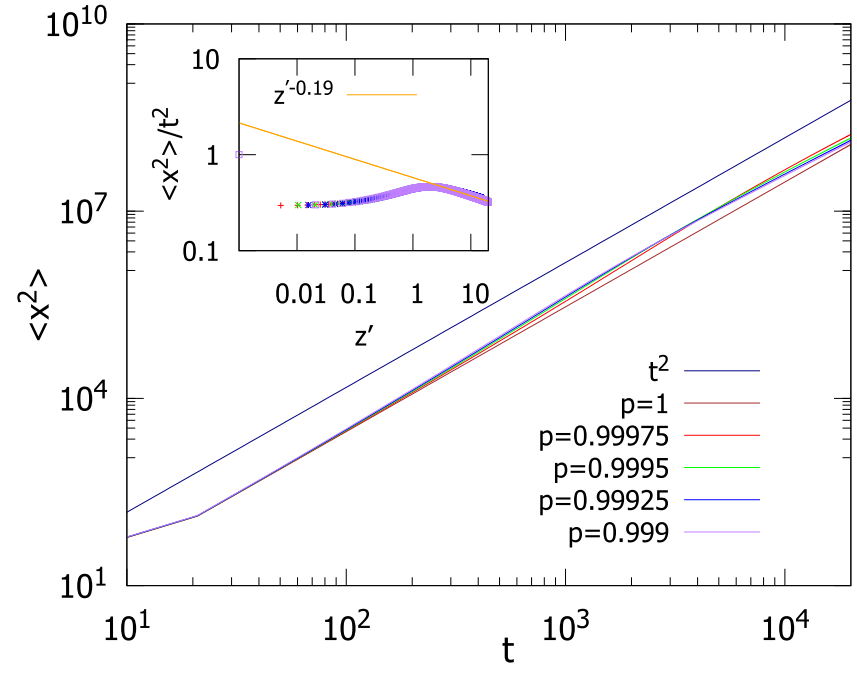

FIG. 6. Scheme I: The unscaled data for $\left\langle x^{2}\right\rangle$ plotted against time $t$. The data for $p=1$ and a curve with quadratic variation are shown for comparison. Inset shows the data collapse obtained on plotting $\left\langle x^{2}\right\rangle / t^{2}$ against the scaling variable $z^{\prime}=(1-p) t$. The solid line has a slope of $\approx-0.19$.

the power law appears beyond time $t=\tau^{\prime} \approx(1-p)^{-1}$ where the scaling function is no longer a constant. This is therefore interpreted as the crossover behavior as also observed for $p \rightarrow$ 0 . The variation of the scaling function beyond $\tau^{\prime}$, however, is nonmonotonic such that fitting a simple functional form is difficult (unlike the case close to $p=0$ ). For very large values of $z^{\prime}$ a power-law form may be fitted. But the exponent has a systematic dependence on the range of $z^{\prime}$ for which the fitting is done; it increases as the estimate is made from later time regimes. Consistent with this, in the inset of Fig. 4, the power-law fitting for a comparatively earlier range of time is shown which corresponds to $v \approx 1.8$. It is expected that if one observes the walk for even larger timescales (difficult computationally), then the exponent value will converge. We conjecture that $v$ will converge to the value 1.5 asymptotically for $p=1-0^{+}$, as in Fig. 5, a sudden increase in the value of $v$ is noted for $p \rightarrow 1$, which suggests that it is actually a discontinuous jump.

Hence we note crossover behavior both close to $p=1$ and $p=0$, which is indicated by the existence of crossover times $\tau$ and $\tau^{\prime}$, which diverge at the two extreme limits with identical exponent 1 .

We note therefore that the walk shows a superdiffusive behavior but with a nonuniversal exponent for small $p$ where the antipersistent effect is strong. For larger values of $p$, the walker behaves as that with random step lengths and persistence is apparently merely the tool that provides the stochasticity. Since it was found in Ref. [9] that the slightest randomness alters the exponent to $3 / 2$, it is not surprising to see that for large $p \neq 1$, one gets the same exponent.

\section{B. Scheme II}

This scheme represents a variation of the first where the walker is either persistent with probability $p$ or with probability $(1-p)$ can choose step length $l=1$ or 2 with probability 

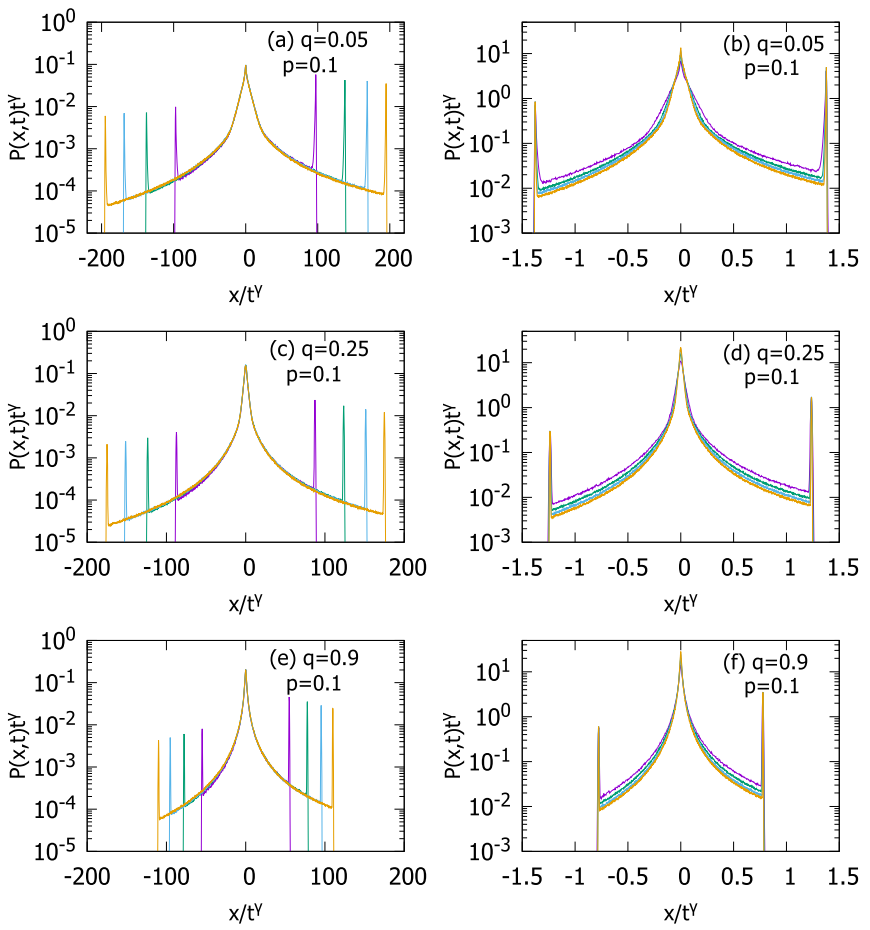

FIG. 7. Scheme II: Data collapse of rescaled $P(x, t)$ using $\gamma=$ 0.5 (left column) and $\gamma=1.0$ (right column). $\gamma=1$ collapse is less sharp.

$q$ and $(1-q)$, respectively. Obviously, for $p=1$ it is always persistent and one recovers an ordinary quantum walk. For $p=0$, it takes step lengths 1 and 2 randomly unless $q=0$ or 1. In fact, if $q=0$ or 1 , then the walk becomes of unique step length eventually (independent of $p$ ); if $q=1$, then that length is 1 and 2 for $q=0$.

Effectively, the total persistence probability $p^{\prime}$ of the walker in this scheme is either $p+q(1-p)$ or $p+(1-$ $q)(1-p)$ at a given time step and it is antipersistent with probability $1-p^{\prime}$. When $q=0.5$, the walker is thus persistent with a probability $p^{\prime}=p / 2+1 / 2$ independent of $q$, and the results should correspond to those obtained for a persistence probability $p^{\prime}$ in Scheme I. Since $p^{\prime}>p_{c}$, one can expect that the results for Scheme II will be identical to Scheme I with the second moment scaling as $t^{3 / 2}$ asymptotically for all $p \geqslant 0$, $q=0.5$. Even when $q \neq 0.5$, the walk is persistent with probability $p / 2+1 / 2$ on an average and antipersistent otherwise. Thus one can expect that it will again be equivalent to Scheme I (with persistence probability $p^{\prime}$ ), when ensemble average is taken if the fluctuation is negligible. In the following we discuss the results which confirm the above picture.

Once again we obtain the distribution $P(x, t)$ which shows a peak centered at the origin and two ballistic peaks. As seen for Scheme I, data collapse is observed with $\gamma=0.5$ for the central peak and $\gamma=1.0$ for the ballistic peaks (Fig. 7) indicating two distinct scaling behaviors $x \propto \sqrt{t}$ and $x \propto t$, respectively.

Next we show the variation of the second moment in Fig. 8 against time which shows the unique exponent $3 / 2$ asymptotically for all $0<p<1$. Here we have plotted the results for several values of $p, q$ showing no significant difference. The

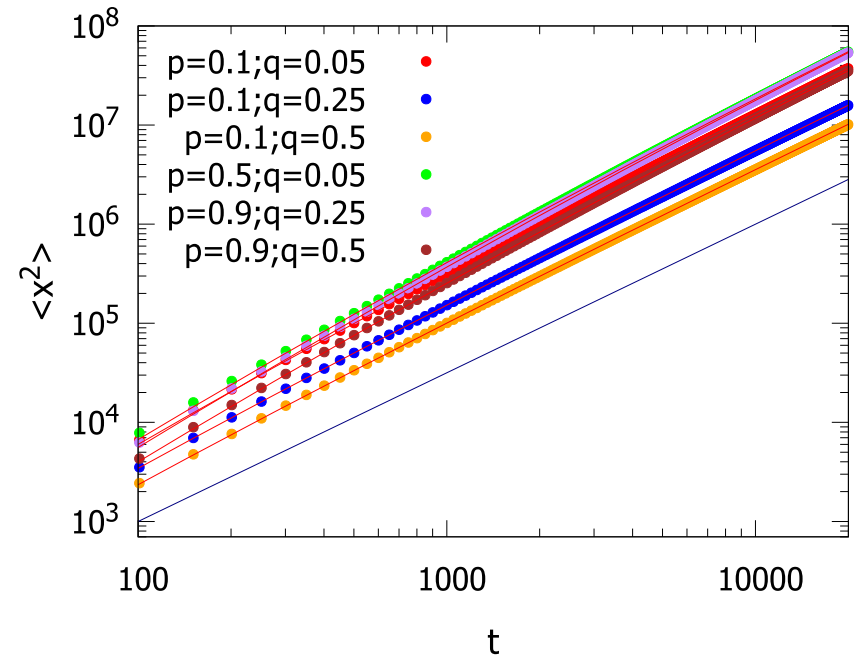

FIG. 8. Scheme II: The second moment for a combination of values of $p$ and $q$. The best-fit curves obtained using Eq. (5) are also shown. The continuous curve has a slope equal to 1.5 .

exponent $v$ as a function of $p$ for $q=0.5$ and a different value of $q$ plotted in Fig. 5 shows that it is $3 / 2$ for the entire region $0<p<1$, confirming that it is equivalent to Scheme I when the persistent probability for the latter is above $p_{c}$. The values of $v$ are obtained by fitting $\left\langle x^{2}\right\rangle$ to the form given in Eq. (5).

\section{SUMMARY AND DISCUSSIONS}

In the present work we have reported the results on a nonMarkovian quantum walk where the step lengths are binary at each time step. It is non-Markovian in the sense that the walker remembers the step length taken in the previous step and tends to repeat it with probability $p$. Thus the choice of the step length is entirely determined by the value of $p$. We numerically evaluate the time evolution of the walk and calculate the distribution $P(x, t)$ and its moments. Scheme I, which is the case when it is strictly antipersistent with probability $(1-p)$, leads to some nonintuitive results when $p$ is small. Precisely, we find how the asymptotic behavior of the second moment changes as $p$ is varied. One can locate four different phases through which $v$ changes. The first is the point $p=0$, where the walk is periodic and correlated over infinite time range and $v=2$. At $p=0$, there is, however, a finite discontinuity in $v$ as $v=1$ for $p=0^{+}$. For $0<p<p_{c}, v$ shows a continuous increase with $p$. Here the walk deviates from its periodic nature and the step lengths are, e.g., 1, 2, 1, 2, . ., 1, 2, 2, 1, 2, 1, . ., 2, 1, 1, 2, 1, 2.. , etc., such that it has two "opposite" strings of variable length repeating alternately. However, the long-time correlation is weakened. As $p$ increases, the step lengths tend to repeat, however, none of the strings, either, e.g., $1,1,1, \ldots, 1$ or $1,2,1,2,1,2$ can have a very large length when $p$ is not equal to 1 or zero. As a result, one gets approximately a random sequence of step lengths. In fact at $p=0.5$ it is purely random. This randomness continues to dominate unless $p$ is exactly equal to 1 so that we get $v=3 / 2$ for $p_{c}<p<1$ and $v=2$ again at $p=1$. 
The result for the region $0<p<p_{c}$ is perhaps the most interesting where we find a $p$-dependent value of $v<3 / 2$ that indicates that the localization is stronger compared to a random choice of the step lengths. The antipersistence effect here is able to confine the walk to a narrower region.

Let us further review the situation close to the extreme values of $p$ regarding the step lengths 1 and 2 as up/down states of a Ising spin. The two sequences $1,2,1,2, \ldots$, and $2,1,2,1, \ldots$, are like antiferromagnetic patterns and are equivalent to simply spin flipped versions of each other in this picture. Hence close to $p=0$ we have two alternating antiferromagnetic patterns and close to $p=1$ we will have two alternating ferromagnetic patterns separated by domain boundaries. Interestingly, for $p \rightarrow 0$ and $p \rightarrow 1$ the exponent $v$ has different values, indicating the antiferromagnetic patterns are responsible for a stronger confinement of the walk. For close to $p=1$, the confinement comes only from the fact that the repetition of the two ferromagnetic patterns is in no way periodic in nature. However, why the antiferromagnetic and ferromagnetic sequences lead to different scaling behavior and why $p_{c}$ is close to $1 / 3$ remain issues to be resolved. One other result is that in general the first moment shows a scaling $\langle x\rangle \propto t^{\nu-1}$ which is not quite obvious. However, this is a result of less importance as the behavior of the first moment is strictly dependent on the symmetry of the initial state.

The second important result we obtain is the crossover phenomena near $p=0$ and 1. Simple power-law scalings for the moments are not possible here as clearly the behavior changes in time. Of course, one can continue the numerical evolution for even larger number of time steps and extract the asymptotic variation. In practice, it is beyond the computational capacity to do so. However, identification of the scaling variable and consequently obtaining a form of the scaling function could help in calculating the asymptotic exponent. We could detect the presence of timescales which diverge at the extreme limits and in the process reveal that the crossover phenomena take place here with a diverging timescale. This divergence signifies that although the exponent $v$ changes discontinuously as $p \rightarrow 0$ or $p \rightarrow 1$, the change can be observed only after long timescales. Away from the extreme limits, the crossover effect becomes less conspicuous.

The results for the persistent quantum walker shows that it is clearly different from the case of random choice of step lengths as long as antipersistence is strong, resulting in a nonuniversal value of the exponent. This is all the more evident from the results of Scheme II in which the effective persistence probability corresponds to that in Scheme I with $p>1 / 2$ and hence corresponds to the random case with $v=3 / 2$. Although the scaling exponent $v$ is $p$ dependent for $p<p_{c}$, the $P(x, t)$ data show collapse with the same type of rescaling as in the random case.

\section{ACKNOWLEDGMENT}

S.M. is grateful for the opportunity to work in the Department of Physics, University of Calcutta. P.S. is grateful to SERB scheme (Government of India) number EMR/2016/005429.
[1] Y. Aharonov, L. Davidovich, and N. Zagury, Phys. Rev. A 48, 1687 (1993).

[2] V. Kendon, Math. Struct. Comput. Sci. 17, 1169 (2007).

[3] J. P. Keating, N. Linden, J. C. F. Matthews, and A. Winter, Phys. Rev. A 76, 012315 (2007).

[4] Y. Yin, D. E. Katsanos, and S. N. Evangelou, Phys. Rev. A 77, 022302 (2008).

[5] P. W. Anderson, Phys. Rev. 109, 1492 (1958).

[6] A. Schreiber, K. N. Cassemiro, V. Potoček, A. Gábris, I. Jex, and C. Silberhorn, Phys. Rev. Lett. 106, 180403 (2011).

[7] T. A. Brun, H. A. Carteret, and A. Ambainis, Phys. Rev. Lett. 91, 130602 (2003).

[8] T. A. Brun, H. A. Carteret, and A. Ambainis, Phys. Rev. A 67, 032304 (2003).
[9] P. Sen, Physica A 514, 266 (2019).

[10] P. Sen, Physica A 545, 123529 (2020).

[11] S. Das, S. Mal, A. Sen(De), and U. Sen, Phys. Rev. A 99, 042329 (2019).

[12] M. McGettrick, Quantum Inf. Comput. 10, 0509 (2010).

[13] P. P. Rohde, G. K. Brennen, and A. Gilchrist, Phys. Rev. A 87, 052302 (2013).

[14] D. Li, M. Mc Gettrick, F. Gao, J. Xu, and Q.-Y. Wen, Phys. Rev. A 93, 042323 (2016).

[15] G. Di Molfetta, D. O. Soares-Pinto, and S. M. Duarte Queirós, Phys. Rev. A 97, 062112 (2018).

[16] M. A. Pires, G. Di Molfetta, and S. M. Duarte Queirós, Sci. Rep. 9, 19292 (2019). 